\title{
Effect of Suvorexant on Nocturnal Delirium in Elderly Patients with Alzheimer's Disease: A Case-series Study
}

\author{
Tomoki Hanazawa $^{1,2}$, Yoshito Kamijo ${ }^{1}$ \\ ${ }^{1}$ Emergency Medical Center and Poison Center, Saitama Medical University Hospital, Saitama, ${ }^{2}$ Department of General Medicine, Fujimi \\ Hospital, Tokyo, Japan
}

\begin{abstract}
Suvorexant, an orexin receptor antagonist used for insomnia, has been shown to have a preventive effect on delirium in a randomized placebo-controlled trial. However, its effectiveness in the management of nocturnal delirium has not yet been determined. Here we report four cases in which elderly patients with moderate to severe Alzheimer's disease who developed nocturnal delirium were treated with suvorexant. In case 1, $15 \mathrm{mg}$ suvorexant was initiated to manage nocturnal delirium refractory to antipsychotics, antidepressants, and a Japanese herbal medicine, resulting in immediate sleep improvement. However, treatment discontinuation led to recurrence of symptoms, which were reversed by recommencing suvorexant. In case 2, as antipsychotics used for the treatment of nocturnal delirium were ineffective, $15 \mathrm{mg}$ suvorexant was administered. The patient achieved rapid improvement in sleep. In case 3, the use of atypical antipsychotics for the treatment of nocturnal delirium was contraindicated, as the patient had diabetes. Therefore, 15 mg suvorexant was administered following good outcomes in cases 1 and 2, resulting in immediate sleep improvement. Finally, in case 4, $15 \mathrm{mg}$ suvorexant was used as an initial medication for nocturnal delirium, and the patient showed sleep improvement immediately. Elevated orexin levels in the cerebrospinal fluid are reportedly linked to sleep deterioration in patients with moderate to severe Alzheimer's disease. The immediate and reproducible action and effectiveness of suvorexant observed in our patients suggest that enhanced cerebral orexin activity might be associated with sleep-wake cycle disturbances due to delirium in elderly patients with Alzheimer's disease.
\end{abstract}

KEY WORDS: Suvorexant; Orexins; Delirium; Alzheimer disease.

\section{INTRODUCTION}

Orexin is an important neurotransmitter that promotes wakefulness. Suvorexant (Belsomra ${ }^{\circledR}$; Merck \& Co., Kenilworth, NJ, USA) induces sleep by selectively and reversibly antagonizing two types of orexin receptors (OX1R and OX2R) [1]. This drug was released for use in Japan in 2014 and in the United States in 2015 with the indication of insomnia management. Recently, a randomized placebo-controlled trial has reported that suvorexant has a preventive effect on delirium [2]. Subjects in this trial received suvorexant or placebo for three nights after be-

Received: April 11, 2018/ Revised: May 8, 2018

Accepted: May 15, 2018

Address for correspondence: Tomoki Hanazawa

Emergency Medical Center and Poison Center, Saitama Medical

University Hospital, 38 Morohongo, Moroyama, Iruma-gun,

Saitama 350-0495, Japan

E-mail: tomokihanazawa34@yahoo.co.jp

ORCID: https://orcid.org/0000-0002-2738-3297 ing newly admitted to a medical hospital. Six of 36 placebo-treated subjects developed delirium during these three days, whereas none of 36 suvorexant-treated subjects did. However, no reports have described the direct effect of suvorexant on delirium.

Here we report four cases in which suvorexant was administered to adjust the sleep-wake cycle in elderly patients with Alzheimer's disease (AD) who developed nocturnal delirium.

\section{CASE}

\section{Case 1}

An 82-year-old man was diagnosed with severe AD five years prior to presentation, but had not taken any AD medication. He was hospitalized in the nearest hospital with a diagnosis of acute postrenal failure due to a penile abscess, for which a vesical fistula was constructed.

(ㄷ) This is an Open-Access article distributed under the terms of the Creative Commons Attribution Non-Commercial License (http://creativecommons.org/licenses/by-nc/4.0) which permits unrestricted non-commercial use, distribution, and reproduction in any medium, provided the original work is properly cited. 
During hospitalization, his state of consciousness dramatically varied over the course of a day with signs of agitation at night. He was started on daily $150 \mathrm{mg}$ quetiapine powder. However, he showed no remarkable improvement. On day 65 of admission, he was transferred to our hospital. From the time of admission, he was often found yelling in a loud voice and attempting to climb over the bedrail from evening to night, with particularly marked nighttime agitation. He was diagnosed with nocturnal delirium according to the diagnostic criteria of the Diagnostic and Statistical Manual of Mental Disorders, fifth edition (DSM-5). In addition to non-pharmacological interventions including cognitively stimulating activities, he was administered daily 7.5 g Yokukansan ${ }^{\circledR}$ (Tsumura \& Co., Tokyo) from day 65 of admission. However, due to his persistent nocturnal delirium, he had to be physically restrained during the night and was treated with $0.5 \mathrm{mg}$ risperidone as required. After taking risperidone, he could sleep for approximately 1 to 2 hours but became agitated soon after waking. Quetiapine was discontinued and 25 $\mathrm{mg}$ trazodone hydrochloride at bed time was initiated on day 68. However, his nocturnal delirium remained unchanged. On day 79 , trazodone hydrochloride was discontinued, and instead, $15 \mathrm{mg}$ suvorexant at bed time was administered. He fell asleep within 30 minutes and slept for 5 to 7 hours from that night on. Although Yokukansan was discontinued on day 81, he maintained good condition. Therefore, he was transferred to the caregiving and recuperation ward on day 121 and suvorexant was discontinued on day 132. However, that night, the patient experienced an acute disturbance of spatial and personal orientation, forcibly removed his vesical fistula, and exhibited motor agitation. The decision to restart suvorexant was made on the following day. The patient subsequently slept well from that night on.

\section{Case 2}

A 91-year-old woman was previously diagnosed with moderate AD but had not taken any medication for AD. She had long been prescribed diuretics for chronic heart failure. She was transported to our hospital by ambulance with a complaint of dyspnea and was diagnosed with acute exacerbation of chronic heart failure. After hospitalization, diuretics, angiotensin-converting-enzyme inhibitors, and aldosterone antagonists were administered, resulting in gradual improvement in roughly seven days. On the other hand, from the day of admission, she often yelled out in a loud voice, pulled out her intravenous line and urethral catheter, and attempted to climb over the bedrail, particularly at night. Her state of consciousness also varied over the course of a day. She was diagnosed with nocturnal delirium. As non-pharmacological interventions for 3 days were ineffective, she was started on $1 \mathrm{mg}$ risperidone at bed time on day 3 of admission. Risperidone helped her sleep for approximately 1 hour, but she became agitated soon after waking. On day 10 , risperidone was discontinued and $15 \mathrm{mg}$ suvorexant at bed time was administered. She fell asleep within 30 minutes and slept for at least 6 hours from that night on.

\section{Case 3}

An 87-year-old man was previously diagnosed with severe $A D$ but had not taken any medication for $A D$. He had been managed for type 2 diabetes with three classes of antidiabetic medication. He was transported to our hospital with complaints of the inability to speak clearly and a feeling of weakness. On arrival, his blood glucose level was $40 \mathrm{mg} / \mathrm{dl}$. His symptoms improved after glucose correction. After hospitalization, treatment for diabetes was adjusted accordingly. On day 3, he pulled out his intravenous line, walked around his room, and spoke in a loud voice as if he was talking to someone during the night. The patient was diagnosed with nocturnal delirium. As nonpharmacological interventions for 3 days did not change his conditions, $15 \mathrm{mg}$ suvorexant at bed time was administered. From that night on, he slept well through the night.

\section{Case 4}

A 98-year-old woman was previously diagnosed with moderate $A D$ and sick sinus syndrome, for which both she and her family had refused any treatment. Her daughter had been receiving calls from our staff twice a day, in the morning and evening, regarding the patient's condition. One night, she wandered around in her house, and was found trying to eat cat food from the feeding box. From that night on, she was often observed speaking toward the door as if she was talking to someone. She was diagnosed with nocturnal delirium. We instructed her family to perform cognitively stimulating activities, which did not prove effective. Thus, $15 \mathrm{mg}$ suvorexant at bed time was administered. From that night on, she slept 
soundly through the night.

\section{DISCUSSION}

Elderly patients, particularly those with pre-existing cognitive impairment, often exhibit delirium after hospitalization, which can make ward management difficult [3]. Delirium is also known to worsen prognosis and thus requires appropriate treatment, including pharmacological management if patients present with symptoms that could threaten their safety, or could result in the interruption of essential therapy $[4,5]$. However, there is no established pharmacotherapy for delirium. Therefore, doctors are forced to use medications such as antipsychotics, antidepressants, and Japanese herbal medicines [6,7], although the efficacy of these drugs remarkably varies from patient to patient [8].

Sleep-wake cycle disturbances including insomnia, excessive daytime napping, and disintegration of the expected circadian rhythm frequently trigger delirium in elderly and cognitively impaired patients. They are also described as a characteristic component of delirium [9-11]. Ramelteon is a melatonin receptor agonist developed to treat insomnia. A randomized placebo-controlled trial demonstrated that ramelteon has a preventive effect on delirium [12]. Moreover, some case reports have described improvement of delirium with ramelteon $[13,14]$.

Suvorexant is the world's first orexin receptor antagonist developed for the management of insomnia. In the present report, suvorexant was administered for the purpose of normalizing sleep-wake cycle disturbances observed in patients with delirium. All four patients with nocturnal delirium successfully fell asleep rapidly after administration of suvorexant, suggesting that the resolution of delirium may be related to the effects of suvorexant on sleep dysregulation. In the first case, while antipsychotics, antidepressants, and a Japanese herbal medicine (Yokukansan) showed no effects in treating his nocturnal delirium, suvorexant markedly improved his sleep. Although nocturnal delirium recurred almost immediately following the discontinuation of suvorexant, recommencement reversed this. The effect of suvorexant on nocturnal delirium was thus suggested to be reproducible. In the second case, the use of antipsychotics for seven days was ineffective in the treatment of nocturnal delirium. We administered suvorexant based on its effects in case 1, and immediate improvement was observed in her sleep. The third patient had diabetes and was undergoing blood glucose adjustment, so we avoided using atypical antipsychotics for the treatment of nocturnal delirium, as these drugs are contraindicated for use in patients with diabetes given their hyperglycemic effects. The patient was started on suvorexant, based on its successful use in the first two cases, showing an immediate improvement. In the fourth case, suvorexant was used as an initial drug for nocturnal delirium and, again, the patient's sleep showed immediate improvement. In all four cases, suvorexant drastically resolved delirium symptoms, or at least improved their sleep.

Neurotransmitter-related changes in AD have been suggested to be associated with sleep disturbance [9]. Patients with moderate to severe AD have been reported to show higher mean orexin levels in the cerebrospinal fluid compared to controls, and have more impaired nocturnal sleep compared to controls and patients with mild AD. This suggests that orexinergic output and function might increase with disease progression and increasing severity, possibly due to an imbalance in neurotransmitter networks that regulate the sleep-wake cycle [15]. The medical history of our patients, such as progressive and gradual decline in cognitive function, neuroimaging results including computed tomography of the brain, cognitive tests, and laboratory data all satisfied the DSM- 5 criteria for $\mathrm{AD}$ with a high level of evidence. Hence, the immediate and reproducible action and effectiveness of suvorexant in our patients suggest that enhanced cerebral orexin activity might be associated with sleep-wake cycle disturbances in elderly patients with moderate to severe AD who develop delirium. Interestingly, when we administered suvorexant for the purpose of managing nocturnal delirium in several elderly patients with dementias other than AD, suvorexant had no effects at all. The exact mechanism of action of suvorexant for delirium is still unclear. However, our findings suggest that suvorexant may be a useful alternative for the treatment of nocturnal delirium in elderly patients with $\mathrm{AD}$.

\section{- Conflicts of Interest}

No potential conflict of interest relevant to this article was reported. 


\section{Author Contributions}

Treatment of all patients and writing the manuscript: Tomoki Hanazawa. Supervision and instruction: Yoshito Kamijo.

\section{ORCID}

Tomoki Hanazawa https://orcid.org/0000-0002-2738-3297

Yoshito Kamijo https://orcid.org/0000-0003-0326-6165

\section{REFERENCES}

1. Sutton EL. Profile of suvorexant in the management of insomnia. Drug Des Devel Ther 2015;9:6035-6042.

2. Hatta K, Kishi Y, Wada K, Takeuchi T, Ito S, Kurata A, et al. Preventive effects of suvorexant on delirium: a randomized placebo-controlled trial. I Clin Psychiatry 2017;78:e970e979.

3. Jackson P, Khan A. Delirium in critically ill patients. Crit Care Clin 2015;31:589-603.

4. Fick DM, Steis MR, Waller IL, Inouye SK. Delirium superimposed on dementia is associated with prolonged length of stay and poor outcomes in hospitalized older adults. J Hosp Med 2013;8:500-505.

5. Inouye SK. Delirium in older persons. NEng/J Med 2006;354: 1157-1165.

6. Saito S, Kobayashi T, Osawa T, Kato S. Effectiveness of Japanese herbal medicine yokukansan for alleviating psychiatric symptoms after traumatic brain injury. Psychogeriatrics 2010;10:45-48.
7. Okamoto Y, Matsuoka Y, Sasaki T, Jitsuiki H, Horiguchi J, Yamawaki S. Trazodone in the treatment of delirium. J Clin Psychopharmacol 1999;19:280-282.

8. Friedman JI, Soleimani L, McGonigle DP, Egol C, Silverstein $\mathrm{JH}$. Pharmacological treatments of non-substance-withdrawal delirium: a systematic review of prospective trials. Am J Psychiatry 2014;171:151-159.

9. Kang DW, Lee CU, Lim HK. Role of sleep disturbance in the trajectory of Alzheimer's disease. Clin Psychopharmacol Neurosci 2017;15:89-99.

10. Fitzgerald JM, Adamis D, Trzepacz PT, O'Regan N, Timmons S, Dunne $C$, et al. Delirium: a disturbance of circadian integrity? Med Hypotheses 2013;81:568-576.

11. Maldonado JR. Neuropathogenesis of delirium: review of current etiologic theories and common pathways. Am J Geriatr Psychiatry 2013;21:1190-1222.

12. Hatta K, Kishi Y, Wada K, Takeuchi T, Odawara T, Usui C, et al. Preventive effects of ramelteon on delirium: a randomized placebo-controlled trial. JAMA Psychiatry 2014;71:397-403.

13. Furuya M, Miyaoka T, Yasuda H, Yamashita S, Tanaka I, Otsuka S, et al. Marked improvement in delirium with ramelteon: five case reports. Psychogeriatrics 2012;12:259262.

14. Kimura R, Mori K, Kumazaki H, Yanagida M, Taguchi S, Matsunaga $\mathrm{H}$. Treatment of delirium with ramelton: initial experience in three patients. Gen Hosp Psychiatry 2011;33: 407-409.

15. Liguori C, Romigi A, Nuccetelli M, Zannino S, Sancesario G, Martorana A, et al. Orexinergic system dysregulation, sleep impairment, and cognitive decline in Alzheimer disease. JAMA Neurol 2014;71:1498-1505. 